\title{
Exact solutions of the 3-wave resonant
} interaction equation

Degasperis, Antonio and Lombardo, Sara 2006

MIMS EPrint: 2007.152

Manchester Institute for Mathematical Sciences

School of Mathematics

The University of Manchester

\footnotetext{
Reports available from: http://eprints.maths.manchester.ac.uk/

And by contacting: The MIMS Secretary

School of Mathematics

The University of Manchester

Manchester, M13 9PL, UK
} 


\title{
Exact solutions of the 3-wave resonant interaction equation
}

\author{
Antonio Degasperis $^{\mathrm{a}, \mathrm{b}}$, Sara Lombardo ${ }^{\mathrm{c}, \mathrm{d}, *}$ \\ a Dipartimento di Fisica, Università di Roma "La Sapienza”, Roma, Italy \\ b Istituto Nazionale di Fisica Nucleare, Sezione di Roma, Roma, Italy \\ ${ }^{\mathrm{c}}$ Department of Applied Mathematics, University of Leeds, Leeds, UK \\ ${ }^{\mathrm{d}}$ Department of Mathematics, Vrije Universiteit, Amsterdam, Netherlands
}

Received 19 September 2005; received in revised form 22 November 2005; accepted 8 January 2006

Communicated by J. Lega

\begin{abstract}
The Darboux-Dressing Transformations are applied to the Lax pair associated to the system of nonlinear equations describing the resonant interaction of three waves in $1+1$ dimensions. We display explicit solutions featuring localized waves whose profile vanishes at the spacial boundary $|x|=\infty$, and which are not pure soliton solutions. These solutions depend on an arbitrary function and allow us to deal with collisions of waves with various profiles.
\end{abstract}

(C) 2006 Elsevier B.V. All rights reserved.

Keywords: 3-wave interaction; Solitons; Integrable PDEs

\section{Introduction}

The propagation in $1+1$ dimensions of three resonating waves is modelled by the following system of three coupled PDEs:

$$
D_{n} \chi_{n}=g_{n} \chi_{n+1}^{*} \chi_{n+2}^{*}, \quad n=1,2,3 \bmod (3),
$$

where the three dependent variables $\chi_{n} \equiv \chi_{n}(x, t)$ are generally complex functions of two real independent variables, $x$ (space) and $t$ (time). The three differential operators $D_{n}, n=1,2,3$, in their linear (i.e. left-hand-side) part are first-order and read

$$
D_{n}:=\partial_{t}+c_{n} \partial_{x},
$$

where the three real constants $c_{n}$ are taken all different among themselves. Each $c_{n}$ is the characteristic velocity at which the field $\chi_{n}$ propagates in the space-time region where no interaction takes place. Here and hereafter a subscripted independent variable denotes partial differentiation with respect to it, asterisks denote complex conjugation and all indices are considered modulo 3 . The three constants $g_{n}$ are generally complex and have the significance of coupling constants. Their values depend of course on the physical context to which the 3Wave Resonant Interaction (3WRI) equation applies. It is remarkable, however that, if these coupling constants satisfy certain conditions (see below), then the 3WRI equation (1) is integrable. In this paper we consider only such case and apply Darboux (or "dressing") transformations to construct explicit solutions of the system (1).

The conditions on the complex constants $g_{n}$ which are necessary and sufficient for the integrability of the 3 WRI equation (1) are:

$$
g_{n}=\sigma_{n}\left|g_{n}\right| \exp (i \Gamma), \quad \sigma_{n}^{2}=1, \quad n=1,2,3 .
$$

\footnotetext{
* Corresponding author at: Department of Mathematics, Vrije Universiteit, Amsterdam, Netherlands.

E-mail addresses: antonio.degasperis@ roma1.infn.it (A. Degasperis), sara@ few.vu.nl (S. Lombardo).
} 
Here the three signs $\sigma_{n}$ and the three moduli $\left|g_{n}\right|$ are arbitrary and the condition is that the (also arbitrary) phase $\Gamma$ is common to the three $g_{n}$ 's. It is worth pointing out here that the structure of the system (1) is left unchanged by the transformation

$$
\bar{\chi}_{n}(\bar{x}, \bar{t})=\alpha_{n} \chi_{n}(x, t), \quad \bar{x}=A x+B t, \bar{t}=C x+D t,
$$

where the three arbitrary constants $\alpha_{n}$ are complex and the four constants $A, B, C, D$ are real and arbitrary except for the requirement $A D-B C \neq 0$; indeed it is clear that such transformation only changes the value of the six constants $c_{n}$ and $g_{n}$. It is easily seen that the transformation (4), as it should, takes $g_{n}$ 's satisfying the integrability conditions (3) into different $g_{n}$ 's which satisfy the same conditions. Hereafter we choose the coupling constants to have the expressions

$$
g_{n}=c_{n+1}-c_{n+2}, \quad n=1,2,3 \bmod (3)
$$

in terms of the velocities $c_{n}$ with the implication that $g_{1}+g_{2}+g_{3}=0$. With this choice the Lax pair associated to the system (1) [1] takes a simple look as it reads

$$
\begin{aligned}
& \Psi_{x}=(-i k B+U(x, t)) \Psi, \\
& \Psi_{t}=(i k A+V(x, t)) \Psi,
\end{aligned}
$$

where $A$ and $B$ are two diagonal, real, traceless matrices

$$
\begin{aligned}
& A=\operatorname{diag}\left\{a_{1}, a_{2}, a_{3}\right\}, \quad B=\operatorname{diag}\left\{b_{1}, b_{2}, b_{3}\right\}, \\
& A=A^{*}, \quad B=B^{*}, \quad \operatorname{tr}(A)=\operatorname{tr}(B)=0 .
\end{aligned}
$$

The $3 \times 3$ matrix $\Psi \equiv \Psi(x, t, k)$ is the common solution of the two ODEs (6) while $U(x, t)$ and $V(x, t)$ are off-diagonal matrices whose entries are related to the three wave fields $\chi_{n}(x, t)$ :

$$
U=\left(\begin{array}{ccc}
0 & \chi_{3} & -\chi_{2}^{*} \\
-\chi_{3}^{*} & 0 & \chi_{1} \\
\chi_{2} & -\chi_{1}^{*} & 0
\end{array}\right), \quad V=\left(\begin{array}{ccc}
0 & -c_{3} \chi_{3} & c_{2} \chi_{2}^{*} \\
c_{3} \chi_{3}^{*} & 0 & -c_{1} \chi_{1} \\
-c_{2} \chi_{2} & c_{1} \chi_{1}^{*} & 0
\end{array}\right)
$$

Moreover, the three characteristic velocities $c_{n}$ which appear in (1) are related to the six real constants $a_{n}$ and $b_{n}$ by the relations

$$
c_{n}=\frac{a_{n+1}-a_{n+2}}{b_{n+1}-b_{n+2}}, \quad n=1,2,3 \bmod (3) .
$$

The integrable character of the 3 WRI equation (1), together with (5), follows from the compatibility $\left(\Psi_{x t}=\Psi_{t x}\right)$ of the two linear equations (6).

The system (1), as it models the interaction of three resonating waves, has attracted much attention in the study of nonlinear waves (see for instance [2]). It can be derived in a quite general way via multiscale analysis of a large class of nonlinear wave equations with weak dispersion and nonlinearity (see for instance [3]). As such, it represents a universal model which is able to capture the lowest order corrections, due to nonlinear effects, to linear propagation. It is here appropriate to point out two special properties of the Eq. (1) which make this model peculiar with respect to most of the integrable nonlinear wave equations (f.i. Nonlinear Schrödinger equation, Korteweg-de Vries equation, Sine-Gordon equation and others). The first one is that the system (1) has no (linear) dispersion (see (2)). The second property is that no self-interaction occurs, namely, the forcing of each wave (i.e. the nonhomogeneous term in the propagation equation (1)) is just the product of the (complex conjugated) amplitudes of the other two waves; see the right-hand side of (1). If we assume that the spacial profile of each one of the three waves is well localized in the far past (i.e. as $t \rightarrow-\infty$ ), then these two properties imply that each wave-profile $\chi_{n}$ translates with no deformation with its characteristic velocity $c_{n}$ up to the time it encounters an other wave. Then, for some time, the interaction takes place where two waves (or the three of them) have spacial overlapping, but eventually, in the far future (i.e. as $t \rightarrow+\infty$ ), each wave is again free to move with no deformation with its own characteristic velocity. Generally, the three wave-profiles at $t=-\infty$ are different from those at $t=+\infty$ since the interaction at intermediate time causes a change of the profile. In the $(x, t)$ plane the asymptotic behaviour is therefore expressed by

$$
\lim _{t \rightarrow \pm \infty} \chi_{n}(y+c t, t)=0, \quad c \neq c_{n}, \quad \lim _{t \rightarrow \pm \infty} \chi_{n}\left(y+c_{n} t, t\right)=\chi_{n}^{( \pm)}(y)
$$

where $y$ is the spacial coordinate in the moving reference frame, and the functions $\chi_{n}^{( \pm)}\left(x-c_{n} t\right)$ are the asymptotic field profiles. Of course, the relevant physical problem is computing the three wave-packets in the future, $\chi_{n}^{(+)}(y)$, once the packets $\chi_{n}^{(-)}(y)$ in the past are given. This large time asymptotic behaviour of the three waves may be referred to as "asymptotic freedom". In terms of the spectral data associated to the three functions $\chi_{n}(x, t)$ via the spectral problem $\Psi_{x}=(-i k B+U(x, t)) \Psi$, see $(6 a)$, the spectral components of the asymptotic states $\chi_{n}^{( \pm)}(y)$ may well be on the continuum spectrum (wave-packets) other than on the discrete spectrum (solitons). These phenomena are quite in contrast with the well-known "separation" process occurring for 
dispersive nonlinear waves where only the discrete spectrum components (solitons) show up as $t \rightarrow \pm \infty$, since the continuum spectrum components (wave-packets) separate from solitons, disperse away and vanish (see for instance [4]). The asymptotic freedom behaviour of the three interacting waves may be given a spectral formulation [2], which is however not discussed here.

In Section 2 we collect formulae which provide us with the tool of the Darboux-Dressing Transformation (DDT), while in Section 3 we obtain, via the DDT, explicit solutions of the 3WRI equations (1). In order to show the asymptotic freedom for wavepackets, we deal with explicit solutions which are not pure soliton solutions, this being the main result of this paper. Section 4 provides an outlook with a few remarks.

\section{The Darboux-Dressing transformation}

Let us now turn our attention to the method of construction of explicit solutions of the 3 WRI equation (1). This construction is based on the Darboux transformation of ODEs and since this transformation is well-known [5] we limit our presentation below to a collection of formulae and propositions. To this purpose it is convenient to observe first that, without any loss of generality, we can give the constants $b_{n}$ and $a_{n}, n=1,2,3$ which appear in the Lax pair of equations (6a) and, respectively, (6b), see also (7), the following expressions:

$$
b_{n}=\frac{1}{3}\left(g_{n+1}-g_{n+2}\right), \quad a_{n}=\frac{1}{3}\left(c_{n+1} g_{n+1}-c_{n+2} g_{n+2}\right), \quad n=1,2,3 \bmod (3) .
$$

The validity of this statement follows from the expressions (9) of the characteristic velocities and (5) of the coupling constants, from the relation $b_{1} g_{1}+b_{2} g_{2}+b_{3} g_{3}=0$, which is implied by them, and from rescaling the spectral parameter $k$. As a result, the spectral parameter $k$ has now the dimension of wave-number/velocity. It should be also noticed, for future reference, that the relations (11) can be inverted to yield the expressions

$$
g_{n}=-\left(b_{n+1}-b_{n+2}\right), \quad n=1,2,3 \bmod (3),
$$

which can be used as alternative to (5) with the implication that $b_{n}=-c_{n}+\left(c_{1}+c_{2}+c_{3}\right) / 3$. We then note that both matrices $U$ and $V$ in the Lax pair (6) satisfy the reduction condition

$$
U=-U^{\dagger}, \quad V=-V^{\dagger},
$$

where the dagger denotes hermitian conjugation. This property (13) induces on the solution $\Psi$ of the two equations (6) the corresponding condition

$$
\Psi^{\dagger}\left(x, t, k^{*}\right) \Psi(x, t, k)=C\left(k, k^{*}\right)
$$

where the matrix $C\left(k, k^{*}\right)$ is constant, namely $x$ - and $t$-independent; its value depends of course only on the arbitrary value $\Psi\left(x_{0}, t_{0}, k\right)$ that the solution $\Psi$ takes at a given point $\left(x_{0}, t_{0}\right)$ of the $(x, t)$ plane.

Consider now a second pair of off-diagonal matrices $U^{(0)}(x, t)$ and $V^{(0)}(x, t)$ which satisfy the same skew-Hermitian condition $\left(U^{(0)}\right)^{\dagger}=-U^{(0)},\left(V^{(0)}\right)^{\dagger}=-V^{(0)}$ as $U$ and $V$ (see (13)); their entries, with obvious notation (see (8)), identify the three complex functions $\chi_{n}^{(0)}(x, t), n=1,2,3$. Let $\Psi^{(0)}(x, t, k)$ be a corresponding nonsingular (i.e. with nonvanishing determinant) matrix solution of the Lax pair (6), i.e.

$$
\Psi_{x}^{(0)}=\left(-i k B+U^{(0)}\right) \Psi^{(0)}, \quad \Psi_{t}^{(0)}=\left(i k A+V^{(0)}\right) \Psi^{(0)}
$$

which have the same form (6) with $U$ and $V$ replaced, respectively, by $U^{(0)}$ and $V^{(0)}$. Assume that the initial condition $\Psi^{(0)}\left(x_{0}, t_{0}, k\right)$ is so chosen that the constant matrix $C^{(0)}\left(k, k^{*}\right)$ (see (14)),

$$
C^{(0)}\left(k, k^{*}\right)=\Psi^{(0)}\left(x, t, k^{*}\right) \Psi^{(0)}(x, t, k),
$$

takes the same value of $C\left(k, k^{*}\right)$, i.e. $C^{(0)}\left(k, k^{*}\right)=C\left(k, k^{*}\right)$. If both compatibility conditions, $\Psi_{x t}^{(0)}=\Psi_{t x}^{(0)}$ and $\Psi_{x t}=\Psi_{t x}$, are satisfied, then $\chi_{n}^{(0)}$ and $\chi_{n}, n=1,2,3$, are two different solutions of the same 3WRI equation (1), and the matrix

$$
D(x, t, k)=\Psi(x, t, k)\left(\Psi^{(0)}(x, t, k)\right)^{-1}
$$

satisfies the pair of differential equations

$$
\begin{aligned}
& D_{x}=i k[D, B]+U D-D U^{(0)}, \\
& D_{t}=-i k[D, A]+V D-D V^{(0)} .
\end{aligned}
$$

Moreover, as a consequence of the reduction conditions (14) and (16) with $C=C^{(0)}$, the matrix $D(x, t, k)$ satisfies also the algebraic equation

$$
D^{\dagger}\left(x, t, k^{*}\right) D(x, t, k)=\mathbf{1} .
$$

The proof of these statements is straightforward. 
The definition (17) can be viewed as a transformation of $\Psi^{(0)}$ into $\Psi$,

$$
\Psi(x, t, k)=D(x, t, k) \Psi^{(0)}(x, t, k),
$$

which consequently yields a transformation of $\chi_{n}^{(0)}$ (the bare solution) into $\chi_{n}$ (the dressed solution). Clearly the dressing approach requires in the first place that $\chi_{n}^{(0)}(x, t)$ and $\Psi^{(0)}(x, t, k)$ be explicitly known. The next step is the construction of the transformation matrix $D(x, t, k)$ via the integration of the ODEs (18). This task however is not straightforward since the coefficients of these differential equations depend on the unknown matrices $U$ and $V$; see (18a) and (18b). The way to overcome this difficulty goes through the a priori assignment of the dependence of the transformation matrix $D(x, t, k)$ on the spectral variable $k$.

The simplest instance of this strategy is illustrated by the following proposition (whose proof is a mere exercise): if $D$ is $k$ independent, $D_{k}=0$, then $D$ is diagonal, $[A, D]=0,[B, D]=0$, and is $x$ - and $t$-independent, i.e. $D_{x}=D_{t}=0$. The associated transformation of $U^{(0)}$ is then a gauge transformation, namely $D=G, U=G U^{(0)} G^{-1}$.

In the following we will consider the larger set of $k$-dependent matrices $D(k)$ which (i) have a rational dependence on the complex variable $k$ and (ii) have nonvanishing limit as $k \rightarrow \infty$. As we consider here only rational dependence on $k$ which can be factorized as product of simple-pole terms, we need to deal only with matrices $D(x, t, k)$ which take the following one-pole expression:

$$
D(x, t, k)=\mathbf{1}+\frac{R(x, t)}{k-\alpha},
$$

the matrix $R(x, t)$ being the residue at the pole $k=\alpha$. It is plain that the transformation matrices $D(k)$ whose $k \rightarrow \infty$ limit is a nonsingular matrix, but not necessarily the identity, may be obtained by multiplying the expression (21) by a gauge transformation $G$. The transformation characterized by the matrix (21) has received considerable attention in the literature [5]; we refer to it as Darboux-Dressing Transformation (DDT). Its existence in our setting is proved below by construction.

In general, the way to obtain an explicit expression of the residue matrix $R(x, t)$ depends on whether the pole $\alpha$ is on the real axis, $\alpha=\alpha^{*}$, or not. However, in the case under investigation, it is easily seen that the condition $\alpha=\alpha^{*}$ implies $R(x, t)=0$. Thus we need to consider only the case in which $\alpha$ is not real, $\alpha \neq \alpha^{*}$. The starting point is the requirement that the matrix $D(x, t, k)$ satisfies the algebraic condition (19) and the differential equations (18). The algebraic condition (19) entails the equation

$$
R+\frac{R^{\dagger} R}{\alpha-\alpha^{*}}=0
$$

whose solution is

$$
R(x, t)=\left(\alpha-\alpha^{*}\right) P(x, t),
$$

where the matrix $P(x, t)$ is an Hermitian projector

$$
P^{2}=P, \quad P^{\dagger}=P .
$$

As for the two differential equations (18), replacing $D(x, t, k)$ with its expression

$$
D(x, t, k)=\mathbf{1}+\left(\frac{\alpha-\alpha^{*}}{k-\alpha}\right) P(x, t),
$$

(see (21) and (23)) yields two algebraic and two differential equations. The algebraic relations read

$$
\begin{aligned}
& U=U^{(0)}+i\left(\alpha-\alpha^{*}\right)[B, P], \\
& V=V^{(0)}-i\left(\alpha-\alpha^{*}\right)[A, P],
\end{aligned}
$$

and give the dressed quantities $\chi_{n}(x, t)$, in terms of the bare ones $\chi_{n}^{(0)}(x, t) n=1,2,3$, and the projector $P$ (see below). The two differential equations are

$$
\begin{aligned}
& P_{x}=(\mathbf{1}-P)\left(-i \alpha^{*} B+U^{(0)}\right) P-P\left(-i \alpha B+U^{(0)}\right)(\mathbf{1}-P), \\
& P_{t}=(\mathbf{1}-P)\left(i \alpha^{*} A+V^{(0)}\right) P-P\left(i \alpha A+V^{(0)}\right)(\mathbf{1}-P) .
\end{aligned}
$$

Let $v$ be an eigenvector of $\mathrm{P}$ with unit eigenvalue, and differentiate with respect to $x$ the eigenvalue equation

$$
P v=v, \quad v=\left(\begin{array}{l}
v_{1} \\
v_{2} \\
v_{3}
\end{array}\right) .
$$


By replacing then $P_{x}$ with the right-hand side of (28a), one obtains the equation

$$
(\mathbf{1}-P)\left[v_{x}-\left(-i \alpha^{*} B+U^{(0)}\right) v\right]=0,
$$

which implies that the vector $v_{x}-\left(-i \alpha^{*} B+U^{(0)}\right) v$ belongs to the subspace on which $P$ projects. At this point we note that, since the dimension of $P$ is 3 , we may well assume, without any loss of generality, that this subspace be one-dimensional. Indeed, it is easy to show that if $P$ projects on a two-dimensional subspace, then $\mathbf{1}-P$ projects on a one-dimensional subspace and the only change in the final result is the value of the parameter $\alpha$, which goes into $\alpha^{*}$. Therefore, $P$ projects on the one-dimensional subspace of the vector $v$ :

$$
P(x, t)=\frac{v(x, t) v^{\dagger}(x, t)}{v^{\dagger}(x, t) v(x, t)}
$$

where in this notation we treat vectors as one-column rectangular matrices. This implies, see (30), that the vector $v_{x}-\left(-i \alpha^{*} B+\right.$ $\left.U^{(0)}\right) v$ is proportional to $v$, i.e.

$$
v_{x}-\left(-i \alpha^{*} B+U^{(0)}\right) v=h v .
$$

On the other hand, since the vector $v$ is identified only modulo a scalar factor, which may well be a function of $x$ and $t$, we may choose this factor in such a way that $v$ satisfies this differential equation (32) with $h=0$.

The differential equation (28b) with respect to the variable $t$ can be handled in a similar way. One obtains therefore that the vector $v(x, t)$ satisfies the differential equations

$$
\begin{aligned}
& v_{x}=\left(-i \alpha^{*} B+U^{(0)}\right) v, \\
& v_{t}=\left(i \alpha^{*} A+V^{(0)}\right) v
\end{aligned}
$$

Once the two equations (33) are solved, the DDT transformation matrix $D(x, t, k)$ is finally given explicitly by (25) with (31).

At this point, we conclude that the method of construction of a novel solution $\chi_{n}(x, t)$ of the 3 WRI equation (1), starting from the knowledge of a given solution $\chi_{n}^{(0)}(x, t)$ is explicitly given by (26) with (31) where the vector $v(x, t)$ is given by

$$
v(x, t)=\Psi^{(0)}\left(x, t, \alpha^{*}\right) v_{0} .
$$

Here $\Psi^{(0)}\left(x, t, \alpha^{*}\right)$ is assumed to be a known solution $\Psi^{(0)}(x, t, k)$ of the Lax pair $(15)$, for $k=\alpha^{*}$, while $v_{0}$ is an arbitrary constant vector,

$$
v_{0}=\left(\begin{array}{l}
\gamma_{1} \\
\gamma_{2} \\
\gamma_{3}
\end{array}\right),
$$

where $\gamma_{1}, \gamma_{2}, \gamma_{3}$ are three (arbitrary) complex parameters. With these specifications (see also (12)), the DDT transformation (26) takes the more explicit form

$$
\chi_{n}=\chi_{n}^{(0)}-i\left(\alpha-\alpha^{*}\right) g_{n} \frac{v_{n+1} v_{n+2}^{*}}{\left|v_{1}\right|^{2}+\left|v_{2}\right|^{2}+\left|v_{3}\right|^{2}}, \quad n=1,2,3 \bmod (3) .
$$

\section{Solutions}

In this section we apply the DDT to known solutions $\chi_{n}^{(0)}(x, t)$ of the 3WRI equation (1) to obtain other solutions of the same equation. Let us consider first the DDT within the class of solutions $\chi_{n}(x, t)$ of the 3 WRI system (1) which are well localized at all times. This class is left invariant by the DDT if the functions $\chi_{n}(x, t)$ vanish sufficiently fast for large $|x|$ at fixed $t, \lim _{x \rightarrow \pm \infty} \chi_{n}(x, t)=0$ (for instance, if they are in $L_{1}(R)$ as functions of the $x$ variable). The simplest choice of the bare solution $\chi_{n}^{(0)}(x, t)$ in this class is, of course, the vanishing one, i.e. $\chi_{n}^{(0)}(x, t)=0$. The corresponding solution which is obtained by applying to it the DDT described in the previous section is the well-known one-soliton solution (see below). The more general class of solutions we compute here originates from the simple observation that even asking that only two of the three fields $\chi_{1}, \chi_{2}$ and $\chi_{3}$ are vanishing yields a solution, which depends moreover on an arbitrary complex function of one real variable. Since the nonvanishing field can be any one of the three, we introduce three different bare solutions as distinguished by the index $j$ :

$$
\chi_{n}^{(0, j)}(x, t)=\delta_{j n} f\left(x_{n}\right), \quad j=1,2,3,
$$


where $f(y)$ is an arbitrary complex function of the real variable $y$ and the three variables $x_{n}, n=1,2,3$, are the characteristic coordinates

$$
x_{n} \equiv x-c_{n} t, \quad n=1,2,3 .
$$

Remark. The distinction of the three solutions (37) by the index $j$ makes sense if the labeling of the three fields $\chi_{n}$ by the index $n$ is fixed. From now on, without any loss of generality, we set such labeling by ordering the three characteristic velocities in the following way:

$$
c_{1}<c_{2}<c_{3}
$$

Indeed we remind the reader that all other relevant parameters, namely the coupling constants $g_{n}$, see (5), and the constants $a_{n}$ and $b_{n}$, see (11), which appear in the Lax equations, see (6a) and (6b) with (7), are functions of the $c_{n}$ 's.

The first step in applying the DDT to the seed solution (37) is of course solving the Lax pair of equations (15) for each one of the three cases (with obvious notation, and see (8))

$$
\begin{aligned}
U^{(0,1)} & =\left(\begin{array}{ccc}
0 & 0 & 0 \\
0 & 0 & f\left(x_{1}\right) \\
0 & -f^{*}\left(x_{1}\right) & 0
\end{array}\right), \quad U^{(0,2)}=\left(\begin{array}{ccc}
0 & 0 & -f^{*}\left(x_{2}\right) \\
0 & 0 & 0 \\
f\left(x_{2}\right) & 0 & 0
\end{array}\right), \\
U^{(0,3)} & =\left(\begin{array}{ccc}
0 & f\left(x_{3}\right) & 0 \\
-f^{*}\left(x_{3}\right) & 0 & 0 \\
0 & 0 & 0
\end{array}\right), \quad V^{(0, j)}=-c_{j} U^{(0, j)},
\end{aligned}
$$

which correspond to the three bare solutions (37). Because of the particular structure of the matrices $U^{(0, j)}$, see $(40)$, and of their dependence on $x$ and $t$ through the characteristic coordinates, see (38), the matrix solution $\Psi^{(0, j)}(x, t, k)$ takes the form

$$
\Psi^{(0, j)}(x, t, k)=\Phi^{(j)}\left(x_{j}, k\right) \exp \left[i \frac{k}{2}\left(b_{j} x-a_{j} t\right)\left(\mathbf{1}-3 P_{j}\right)\right],
$$

where the three matrices $P_{j} \quad j=1,2,3$, are the three diagonal projectors

$$
P_{1}=\left(\begin{array}{lll}
1 & 0 & 0 \\
0 & 0 & 0 \\
0 & 0 & 0
\end{array}\right), \quad P_{2}=\left(\begin{array}{lll}
0 & 0 & 0 \\
0 & 1 & 0 \\
0 & 0 & 0
\end{array}\right), \quad P_{3}=\left(\begin{array}{lll}
0 & 0 & 0 \\
0 & 0 & 0 \\
0 & 0 & 1
\end{array}\right)
$$

Solving the equations of the Lax pair now reduces to solving only the $3 \times 3$ matrix ODE

$$
\Phi_{y}^{(j)}(y, k)=\left[\frac{i}{2} g_{j} k\left(P_{j+1}-P_{j+2}\right)+U^{(0, j)}(y)\right] \Phi^{(j)}(y, k),
$$

which is however easily seen to be equivalent to the standard Zakharov-Shabat system of two equations

$$
\phi_{1 y}=\frac{i}{2} \lambda \phi_{1}+f(y) \phi_{2}, \quad \phi_{2 y}=-\frac{i}{2} \lambda \phi_{2}-f^{*}(y) \phi_{1}
$$

for the two functions $\phi_{1}(y, \lambda)$ and $\phi_{2}(y, \lambda)$. Indeed, once a solution of the system (44) is known, the $3 \times 3$ matrices $\Phi^{(j)}(y, k)$, for $j=1,2,3$, which solve (43) are also known and read

$$
\begin{aligned}
\Phi^{(1)}(y, k) & =\left(\begin{array}{ccc}
1 & 0 & 0 \\
0 & \phi_{1}\left(y, g_{1} k\right) & -\phi_{2}^{*}\left(y, g_{1} k^{*}\right) \\
0 & \phi_{2}\left(y, g_{1} k\right) & \phi_{1}^{*}\left(y, g_{1} k^{*}\right)
\end{array}\right), \\
\Phi^{(2)}(y, k) & =\left(\begin{array}{ccc}
\phi_{2}\left(y, g_{2} k\right) & 0 & -\phi_{1}^{*}\left(y, g_{2} k^{*}\right) \\
0 & 1 & 0 \\
\phi_{1}\left(y, g_{2} k\right) & 0 & \phi_{2}^{*}\left(y, g_{2} k^{*}\right)
\end{array}\right), \\
\Phi^{(3)}(y, k) & =\left(\begin{array}{ccc}
\phi_{1}\left(y, g_{3} k\right) & -\phi_{2}^{*}\left(y, g_{3} k^{*}\right) & 0 \\
\phi_{2}\left(y, g_{3} k\right) & \phi_{1}^{*}\left(y, g_{3} k^{*}\right) & 0 \\
0 & 0 & 1
\end{array}\right) .
\end{aligned}
$$

For future reference, we display also the asymptotic behaviour of the solution of the Zakharov-Shabat system (44),

$$
\phi_{1}(y, \lambda) \underset{y \rightarrow \pm \infty}{\longrightarrow} z_{1}^{( \pm)}(\lambda) \exp \left(\frac{i}{2} \lambda y\right), \quad \phi_{2}(y, \lambda) \underset{y \rightarrow \pm \infty}{\longrightarrow} z_{2}^{( \pm)}(\lambda) \exp \left(-\frac{i}{2} \lambda y\right),
$$


which introduces the four functions $z_{1}^{( \pm)}(\lambda)$ and $z_{2}^{( \pm)}(\lambda)$, two of which can of course be arbitrarily given while the other two can be obtained by integrating the ODEs (44).

Let us now consider the dressed solution by applying the DDT given in the previous section to the bare solution (37). Its expression (see (36)) is

$$
\chi_{n}^{(j)}(x, t)=\delta_{j n} f\left(x_{n}\right)+2 \eta g_{n} \frac{v_{n+1}^{(j)}(x, t) v_{n+2}^{(j) *}(x, t)}{\left|v_{1}^{(j)}(x, t)\right|^{2}+\left|v_{2}^{(j)}(x, t)\right|^{2}+\left|v_{3}^{(j)}(x, t)\right|^{2}},
$$

where the parameter $\eta$ is the imaginary part of the complex pole $\alpha$ which has been introduced in the DDT formula (25), namely

$$
\alpha=\rho+i \eta
$$

According to the definition (34), the three 3-vectors $v^{(j)}(x, t)$, with components $v_{n}^{(j)}(x, t)$, are provided by the formula (see (41))

$$
v^{(j)}(x, t)=\Phi^{(j)}\left(x_{j}, \alpha^{*}\right) \exp \left[i \frac{\alpha^{*}}{2}\left(b_{j} x-a_{j} t\right)\left(\mathbf{1}-3 P_{j}\right)\right] v_{0},
$$

where the arbitrary complex constant vector $v_{0}$ introduces in the solution $\chi_{n}^{(j)}(x, t)$ the three arbitrary parameters $\gamma_{1}, \gamma_{2}$ and $\gamma_{3}$; see (35). This new solution (47) of the 3WRI equation depends on the function $f(y)$ which characterizes the bare solution (37) and its behavior may be roughly described as the interaction of the bare $j$-th wave $f\left(x-c_{j} t\right)$ with soliton-type bumps in the other two waves whose speed may be larger or smaller than $c_{j}$, a behavior which may be quite complicated at finite intermediate times. Here we do not dwell in detailing such behavior at finite times, rather we compute the asymptotic states as $t \rightarrow \pm \infty$ whose profile and properties really matter in an applicative context. According to the general definition (10), we now compute, for each value of $j$, the six functions $\chi_{n}^{(j)( \pm)}(y)$ by performing the limits

$$
\lim _{t \rightarrow \pm \infty} \chi_{n}^{(j)}\left(y+c_{n} t, t\right)=\chi_{n}^{(j)( \pm)}(y) .
$$

This computation is elementary but lengthy, and it is omitted. The relevant results take the following expressions:

$$
\begin{aligned}
& \left\{\begin{array}{l}
\lim _{t \rightarrow s_{j} \infty} \chi_{j}^{(j)}\left(y+c_{j} t, t\right)=f(y)+2 \eta g_{j} \frac{w_{j+1}^{(j)}(y) w_{j+2}^{(j) *}(y)}{\left|w_{j+1}^{(j)}(y)\right|^{2}+\left|w_{j+2}^{(j)}(y)\right|^{2}}, \\
\lim _{t \rightarrow-s_{j} \infty} \chi_{j}^{(j)}\left(y+c_{j} t, t\right)=f(y),
\end{array}\right. \\
& \left\{\begin{array}{l}
\lim _{t \rightarrow s_{j+1} \infty} \chi_{j+1}^{(j)}\left(y+c_{j+1} t, t\right)=2 \eta g_{j+1} \exp \left(i \rho g_{j+1} y\right) \frac{\gamma_{j}^{*} u_{j+2}^{(j)\left(-s_{j}\right)}}{\left|\gamma_{j}\right|^{2} \exp \left(-\eta g_{j+1} y\right)+\left|u_{j+2}^{(j)\left(-s_{j}\right)}\right|^{2} \exp \left(\eta g_{j+1} y\right)}, \\
\lim _{t \rightarrow-s_{j+1} \infty} \chi_{j+1}^{(j)}\left(y+c_{j+1} t, t\right)=0,
\end{array}\right. \\
& \left\{\begin{array}{l}
\lim _{t \rightarrow s_{j+2} \infty} \chi_{j+2}^{(j)}\left(y+c_{j+2} t, t\right)=2 \eta g_{j+2} \exp \left(i \rho g_{j+2} y\right) \frac{\gamma_{j} u_{j+1}^{(j)\left(s_{j}\right) *}}{\left|\gamma_{j}\right|^{2} \exp \left(\eta g_{j+2} y\right)+\left|u_{j+1}^{(j)\left(s_{j}\right)}\right|^{2} \exp \left(-\eta g_{j+2} y\right)}, \\
\lim _{t \rightarrow-s_{j+2} \infty} \chi_{j+2}^{(j)}\left(y+c_{j+2} t, t\right)=0 .
\end{array}\right.
\end{aligned}
$$

The symbols we have introduced here are: the three signs

$$
s_{j}=\operatorname{sign}\left(\eta g_{j}\right), \quad s_{j}^{2}=1, \quad j=1,2,3,
$$

the real parameter $\rho$ which is the real part of the complex pole $\alpha$, see (48), the three $y$-dependent vectors (see (45a), (45b), (45c) and (35))

$$
w^{(j)}(y)=\Phi^{(j)}\left(y, \alpha^{*}\right) v_{0},
$$

and the three constant vectors

$$
u^{(1)( \pm)}=\left(\begin{array}{c}
\gamma_{1} \\
\gamma_{2} z_{1}^{( \pm)}\left(g_{1} \alpha^{*}\right)-\gamma_{3} z_{2}^{( \pm) *}\left(g_{1} \alpha\right) \\
\gamma_{2} z_{2}^{( \pm)}\left(g_{1} \alpha^{*}\right)+\gamma_{3} z_{1}^{( \pm) *}\left(g_{1} \alpha\right)
\end{array}\right)
$$




$$
\begin{aligned}
& u^{(2)( \pm)}=\left(\begin{array}{c}
\gamma_{1} z_{2}^{( \pm)}\left(g_{2} \alpha^{*}\right)-\gamma_{3} z_{1}^{( \pm) *}\left(g_{2} \alpha\right) \\
\gamma_{2} \\
\gamma_{1} z_{1}^{( \pm)}\left(g_{2} \alpha^{*}\right)+\gamma_{3} z_{2}^{( \pm) *}\left(g_{2} \alpha\right)
\end{array}\right), \\
& u^{(3)( \pm)}=\left(\begin{array}{c}
\gamma_{1} z_{1}^{( \pm)}\left(g_{3} \alpha^{*}\right)-\gamma_{2} z_{2}^{( \pm) *}\left(g_{3} \alpha\right) \\
\gamma_{1} z_{2}^{( \pm)}\left(g_{3} \alpha^{*}\right)+\gamma_{2} z_{1}^{( \pm) *}\left(g_{3} \alpha\right) \\
\gamma_{3}
\end{array}\right),
\end{aligned}
$$

where the four parameters $z_{1}^{( \pm)}\left(g_{j} \alpha\right), z_{2}^{( \pm)}\left(g_{j} \alpha\right)$ and $z_{1}^{( \pm)}\left(g_{j} \alpha^{*}\right), z_{2}^{( \pm)}\left(g_{j} \alpha^{*}\right)$, for $j=1,2,3$, are the values that the two functions $z_{1}^{( \pm)}(\lambda)$ and $z_{2}^{( \pm)}(\lambda)$, introduced via the asymptotic behavior (46), take at $\lambda=g_{j} \alpha$ and $\lambda=g_{j} \alpha^{*}$, respectively.

At this point we are in the position to discuss the asymptotic in- and out-states corresponding to our solution. Since we aim to attract the attention of the reader to potential applications, we chose to have the bare wave $f\left(x-c_{j} t\right)$ in the initial state (say as $t \rightarrow-\infty$ ). Therefore, as indicated by the limit formula (51a), we have to set $s_{j}=1$ for each $j$, and this implies, according to the definition (52) and to the signs of the coupling constants, see (5) and (39),

$$
\operatorname{sign}\left(g_{1}\right)=-1, \quad \operatorname{sign}\left(g_{2}\right)=1, \quad \operatorname{sign}\left(g_{3}\right)=-1,
$$

that we pick the pole $\alpha$ of the DDT in the lower half of the complex plane, $\eta<0$, for $j=1$ and $j=3$ and in the upper half of the complex plane, $\eta>0$, for $j=2$. By taking into account that these choices of the sign of the parameter $\eta$ entail that

$$
\begin{cases}s_{2}=-1, \quad s_{3}=1, & \text { for } j=1, \\ s_{1}=-1, \quad s_{3}=-1, & \text { for } j=2, \\ s_{1}=1, \quad s_{2}=-1, & \text { for } j=3,\end{cases}
$$

the final expressions of the asymptotic profiles of the three waves are readily read out of the formulae (51a), (51b) and (51c), and are

$$
\begin{array}{ll}
\chi^{(1)(-)}(x, t)=\left(\begin{array}{c}
f\left(x-c_{1} t\right) \\
S_{2}^{(1)}\left(x-c_{2} t\right) \\
0
\end{array}\right), & \chi^{(1)(+)}(x, t)=\left(\begin{array}{c}
F^{(1)}\left(x-c_{1} t\right) \\
0 \\
S_{3}^{(1)}\left(x-c_{3} t\right)
\end{array}\right), \\
\chi^{(2)(-)}(x, t)=\left(\begin{array}{c}
S_{1}^{(2)}\left(x-c_{1} t\right) \\
f\left(x-c_{2} t\right) \\
S_{3}^{(2)}\left(x-c_{3} t\right)
\end{array}\right), & \chi^{(2)(+)}(x, t)=\left(\begin{array}{c}
F^{(2)}\left(x-c_{2} t\right) \\
0
\end{array}\right), \\
\chi^{(3)(-)}(x, t)=\left(\begin{array}{c}
0 \\
S_{2}^{(3)}\left(x-c_{2} t\right) \\
f\left(x-c_{3} t\right)
\end{array}\right), & \chi^{(3)(+)}(x, t)=\left(\begin{array}{c}
S_{1}^{(3)}\left(x-c_{1} t\right) \\
0 \\
F^{(3)}\left(x-c_{3} t\right)
\end{array}\right) .
\end{array}
$$

In these formulae we have expressed the asymptotic profiles by means of the following expressions, which again are just implied by (51a), (51b) and (51c):

$$
\begin{aligned}
& F^{(j)}(y)=f(y)+2 \eta g_{j} \frac{w_{j+1}^{(j)}(y) w_{j+2}^{(j) *}(y)}{\left|w_{j+1}^{(j)}(y)\right|^{2}+\left|w_{j+2}^{(j)}(y)\right|^{2}}, \\
& \left\{\begin{array}{l}
S_{2}^{(1)}(y)=2 \eta g_{2} \exp \left(i \rho g_{2} y\right) \frac{\gamma_{1}^{*} u_{3}^{(1)(-)}}{\left|\gamma_{1}\right|^{2} \exp \left(-\eta g_{2} y\right)+\left|u_{3}^{(1)(-)}\right|^{2} \exp \left(\eta g_{2} y\right)}, \\
S_{3}^{(1)}(y)=2 \eta g_{3} \exp \left(i \rho g_{3} y\right) \frac{\gamma_{1} u_{2}^{(1)(+) *}}{\left|\gamma_{1}\right|^{2} \exp \left(\eta g_{3} y\right)+\left|u_{2}^{(1)(+)}\right|^{2} \exp \left(-\eta g_{3} y\right)},
\end{array}\right. \\
& \left\{\begin{array}{l}
S_{1}^{(2)}(y)=2 \eta g_{1} \exp \left(i \rho g_{1} y\right) \frac{\gamma_{2} u_{3}^{(2)(+) *}}{\left|\gamma_{2}\right|^{2} \exp \left(\eta g_{1} y\right)+\left|u_{3}^{(2)(+)}\right|^{2} \exp \left(-\eta g_{1} y\right)}, \\
S_{3}^{(2)}(y)=2 \eta g_{3} \exp \left(i \rho g_{3} y\right) \frac{\gamma_{2}^{*} u_{1}^{(2)(-)}}{\left|\gamma_{2}\right|^{2} \exp \left(-\eta g_{3} y\right)+\left|u_{1}^{(2)(-)}\right|^{2} \exp \left(\eta g_{3} y\right)},
\end{array}\right.
\end{aligned}
$$




$$
\left\{\begin{array}{l}
S_{1}^{(3)}(y)=2 \eta g_{1} \exp \left(i \rho g_{1} y\right) \frac{\gamma_{3}^{*} u_{2}^{(3)(-)}}{\left|\gamma_{3}\right|^{2} \exp \left(-\eta g_{1} y\right)+\left|u_{2}^{(3)(-)}\right|^{2} \exp \left(\eta g_{1} y\right)}, \\
S_{2}^{(3)}(y)=2 \eta g_{2} \exp \left(i \rho g_{2} y\right) \frac{\gamma_{3} u_{1}^{(3)(+) *}}{\left|\gamma_{3}\right|^{2} \exp \left(\eta g_{2} y\right)+\left|u_{1}^{(3)(+)}\right|^{2} \exp \left(-\eta g_{2} y\right)} .
\end{array}\right.
$$

Their meaning is quite transparent: the function $F^{(j)}(y)$, which always appears in an out-state, shows how the profile of the incoming wave-packet $f(y)$ changes because of the collision with solitonic waves, while the functions $S_{n}^{(j)}(y)$ show the standard sech-shape of the solitons (see below) appearing in the incoming and/or outgoing states. The three different collision processes described by these asymptotic states, (57a), (57b), (57c), are controlled by various parameters introduced via the DDT, namely the complex pole $\alpha=\rho+i \eta$ and the complex components $\gamma_{j}$ of the vector $v_{0}$ (see (35)) on the one hand, and the (largely arbitrary) complex function $f(y)$ characterizing the bare solution on the other hand. We note moreover that only in the process with $j=2$ there are three nonvanishing waves in the in-state, a feature which is not found in the one-soliton solution (see below). We also note that not only does the deformation $F^{(j)}(y)$ of the initial profile $f(y)$ (see (53)) depend on the solution of the ZS equations (44), but also the position of the in-coming and out-going solitons (see below) depends on these solutions through their asymptotic behavior, see (59a), (59b), (59c) together with (54a), (54b), (54c) and (46).

At this point it is evident that numerical experiments, which do not rely on the integration of the 3WRI system of PDEs (1) but just on analytical explicit expressions, are possible if one chooses the function $f(y)$ as a "solvable potential", namely a function such that the general solution of the ZS equations (44) is explicitly known. There exist indeed many such choices. We just pick one of them here below to produce explicit formulae which one can use to experiment on three-wave interaction processes. Before doing this however we observe that the asymptotic profiles $S_{n}^{(j)}(y)$, see (57a), (57b), (57c) and (59a), (59b), (59c), coincide with the asymptotic states of the one-soliton solution of the 3WRI equation. This solution obtains by applying the DDT to the trivial choice $f(y)=0$ and by using the formulae given above. In this particular case the index $j$ which we use to distinguish three different initial bare solutions (see (37)) is, of course, useless and it is dropped. The resulting expression of the one-soliton solution then reads, in our notation,

$$
\begin{aligned}
\chi_{n}(x, t)= & 2 \eta g_{n} \exp \left(i \rho g_{n} x_{n}\right) \\
& \times \frac{\gamma_{n+1} \gamma_{n+2}^{*}}{\left|\gamma_{n+1}\right|^{2} \exp \left(\eta g_{n} x_{n}\right)+\left|\gamma_{n+2}\right|^{2} \exp \left(-\eta g_{n} x_{n}\right)+\left|\gamma_{n}\right|^{2} \exp \left[\eta\left(g_{n+2}-g_{n+1}\right) x_{n}\right] \exp \left(-2 \eta g_{n+1} g_{n+2} t\right)},
\end{aligned}
$$

where the signs $s_{n}, n=1,2,3$, are defined by (52) with the order convention (39). The large time limits of this solution are readily found to be

$$
\begin{aligned}
& \chi_{n}\left(y+c_{n} t, t\right) \underset{t \rightarrow-s_{n} \infty}{\longrightarrow} 0, \\
& \chi_{n}\left(y+c_{n} t, t\right) \underset{t \rightarrow s_{n} \infty}{\longrightarrow} 2 \eta g_{n} \exp \left(i \rho g_{n} y\right) \frac{\gamma_{n+1} \gamma_{n+2}^{*}}{\left|\gamma_{n+1}\right|^{2} \exp \left(\eta g_{n} y\right)+\left|\gamma_{n+2}\right|^{2} \exp \left(-\eta g_{n} y\right)},
\end{aligned}
$$

and this last expression coincides, apart from obvious replacement of the parameters $\gamma$ 's, with the general expressions (51a) with $f(y)=0$ or (51b) or (51c). If we choose moreover $\eta>0$, it is easily seen that, because of (55), the asymptotic states here correspond to the expressions given above for $j=2$ and $f(y)=0$. These states may be given also the familiar sech form

$$
\begin{aligned}
& \left\{\begin{array}{l}
\chi_{1}^{(-)}(y)=\frac{\eta g_{1} \exp \left[i\left(\rho g_{1} y+\theta_{2}-\theta_{3}\right)\right]}{\cosh \left[\eta g_{1}\left(y-\xi_{1}\right)\right]}, \\
\chi_{2}^{(-)}(y)=0, \\
\chi_{3}^{(-)}(y)=\frac{\eta g_{3} \exp \left[i\left(\rho g_{3} y+\theta_{1}-\theta_{2}\right)\right]}{\cosh \left[\eta g_{3}\left(y-\xi_{3}\right)\right]},
\end{array}\right. \\
& \left\{\begin{array}{l}
\chi_{1}^{(+)}(y)=0, \\
\chi_{2}^{(+)}(y)=\frac{\eta g_{2} \exp \left[i\left(\rho g_{2} y+\theta_{3}-\theta_{1}\right)\right]}{\cosh \left[\eta g_{2}\left(y-\xi_{2}\right)\right]}, \\
\chi_{3}^{(+)}(y)=0,
\end{array}\right.
\end{aligned}
$$

by introducing the phases $\theta_{n}$ and the positions $\xi_{n}$ via the relation

$$
\gamma_{n}=\exp \left(i \theta_{n}\right) \exp \left[\frac{\eta}{3}\left(g_{n+1} \xi_{n+1}-g_{n+2} \xi_{n+2}\right)\right]
$$


together with the condition

$$
g_{1} \xi_{1}+g_{2} \xi_{2}+g_{3} \xi_{3}=0 .
$$

Moreover, we observe that the asymptotic soliton, whose generic expression is

$$
S(y)=2 \eta g \exp (i \rho g y) \frac{\gamma^{(+)} \gamma^{(-) *}}{\left|\gamma^{(+)}\right|^{2} \exp (\eta g y)+\left|\gamma^{(-)}\right|^{2} \exp (-\eta g y)},
$$

may be characterized in the usual way by its amplitude $A=E / 2$, width $\lambda=4 / E$ and position $\xi=\log \left(\left|\gamma^{(-)} / \gamma^{(+)}\right|\right) /(\eta g)$ where $E$ is the "energy"

$$
E=\int_{-\infty}^{+\infty} \mathrm{d} y|S(y)|^{2}=2|\eta g| .
$$

Let us now look at one explicit solution. To this aim we choose the undressed wave-profile

$$
f(y)=a \exp (-q|y|)
$$

where $a$ and $q$ are arbitrary real parameters $\left(a=a^{*}, q=q^{*}\right)$ and $q$ is positive, $q>0$. The chain of steps we have to make to arrive at the asymptotic state expressions (57a), (57b), (57c) is the following: (i) solving the ZS equations (44) and obtaining therefore also the expressions of $z_{1}^{( \pm)}(\lambda)$ and $z_{2}^{( \pm)}(\lambda)$, see (46), (ii) computing the vector functions $w^{(j)}(y)$, see (53) and (45a), (45b), (45c), (iii) computing the constant vector $u^{(j)( \pm)}$, see (54a), (54b), (54c), and finally (iv) using the expressions (58) together with (59a), (59b), (59c). These steps can be made by using the properties of Bessel functions $J_{v}(z)$ (see for instance [6]), and we report here only the resulting expressions with the following notation:

$$
\begin{aligned}
& \zeta=\frac{a}{q}, \quad z=\zeta \exp (-q|y|), \quad v_{ \pm}=\frac{1}{2} \pm \frac{i \lambda}{2 q}, \\
& C_{ \pm}=J_{-v_{ \pm}}^{2}(\zeta)-J_{\nu_{\mp}}^{2}(\zeta) .
\end{aligned}
$$

The solution of the ZS equations (44) we choose is

$$
\begin{aligned}
\phi_{1}(y, \lambda)= & \exp \left(-\frac{q}{2}|y|\right)\left\{H(y)\left[C_{-} J_{v_{-}}(\zeta) J_{-v_{+}}(z)-C_{+} J_{-v_{-}}(\zeta) J_{v_{+}}(z)\right]\right. \\
& \left.+H(-y)\left[C_{-} J_{-v_{+}}(\zeta) J_{v_{-}}(z)-C_{+} J_{v_{+}}(\zeta) J_{-v_{-}}(z)\right]\right\}, \\
\phi_{2}(y, \lambda)= & \exp \left(-\frac{q}{2}|y|\right)\left\{H(y)\left[C_{-} J_{v_{-}}(\zeta) J_{v_{-}}(z)+C_{+} J_{-v_{-}}(\zeta) J_{-v_{-}}(z)\right]\right. \\
& \left.+H(-y)\left[C_{-} J_{-v_{+}}(\zeta) J_{-v_{+}}(z)+C_{+} J_{v_{+}}(\zeta) J_{v_{+}}(z)\right]\right\},
\end{aligned}
$$

where $H(y)$ is the standard Heaviside step function $(H(y)=1$ if $y \geq 0$ and $H(y)=0$ if $y<0)$. These expressions of $\phi_{j}(y, \lambda)$ entail that the asymptotic constants (see (46)) may be chosen as

$$
\begin{aligned}
& z_{1}^{( \pm)}= \pm\left(\frac{2}{\zeta}\right)^{v_{ \pm}} J_{v_{\mp}}(\zeta) C_{\mp} / \Gamma\left(v_{\mp}\right), \\
& z_{2}^{( \pm)}=\left(\frac{2}{\zeta}\right)^{v_{\mp}} J_{-v_{\mp}}(\zeta) C_{ \pm} / \Gamma\left(v_{ \pm}\right),
\end{aligned}
$$

where $\Gamma$ denotes the Gamma function (see for instance [6]). From these expressions all other relevant quantities can be computed by simple algebra. For the benefit of the reader we report here the standard properties of the Bessel and Gamma functions which have been used to derive these expressions:

$$
\begin{aligned}
& J_{v}(z)=\left(\frac{z}{2}\right)^{v}\left[\frac{1}{\Gamma(v+1)}+O\left(z^{2}\right)\right], \\
& z \Gamma\left(v_{+}\right) \Gamma\left(v_{-}\right)\left[J_{v_{+}}(z) J_{v_{-}}(z)+J_{-v_{+}}(z) J_{-v_{-}}(z)\right]=2,
\end{aligned}
$$

the first one of which showing the leading term of $J_{v}(z)$ for small $|z|$.

It is now rather straightforward to play with the various parameters, $a, q, \rho, \eta, \gamma_{n}$ and $c_{n}$, together with the index $j=1,2,3$, to explore the interaction of the picked wave-profile $f(y)=a \exp (-q|y|)$, see (67), with solitons in a variety of different regimes with respect to relative velocities, energies and soliton positions. A few examples of asymptotic in- and out-state profiles for such processes are graphically displayed in the following figures. These examples have been chosen to display processes which may be of interest in potential applications of our formulae. They show the effect of collision with one soliton (for $j=1$, see Fig. 1) 

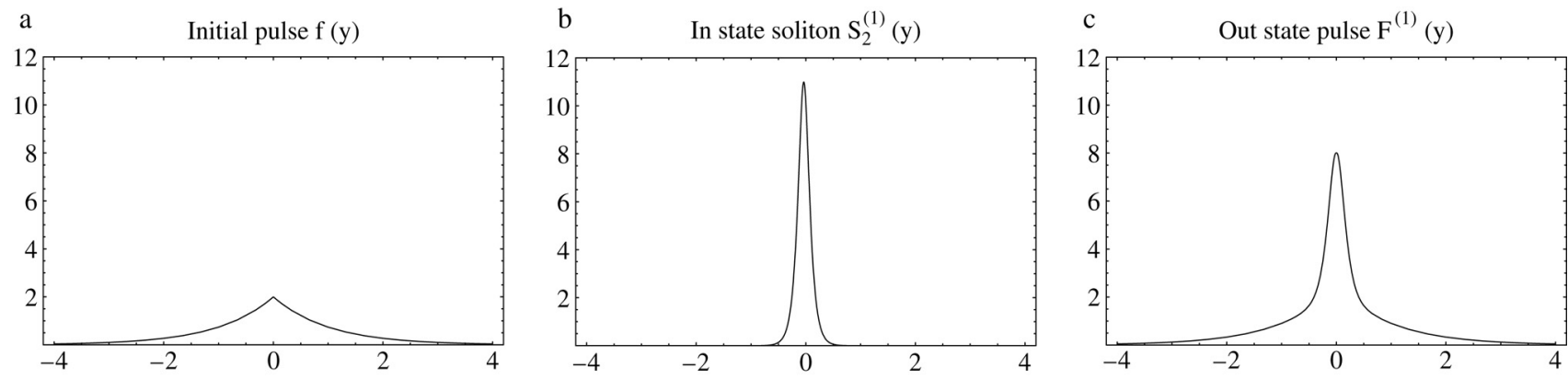

Fig. 1. Amplitude amplification.
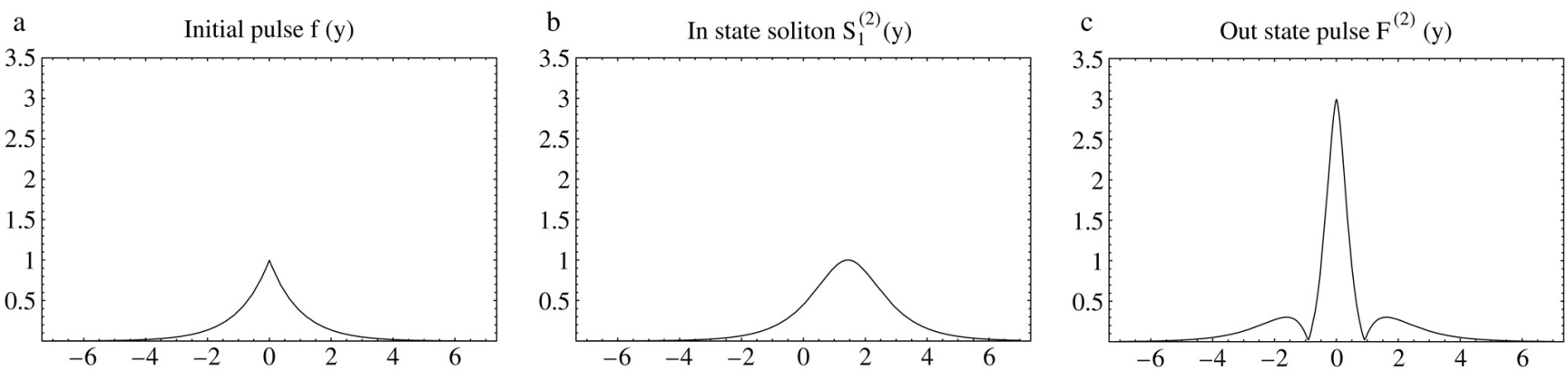

Fig. 2. Width-narrowing and amplitude-amplification.
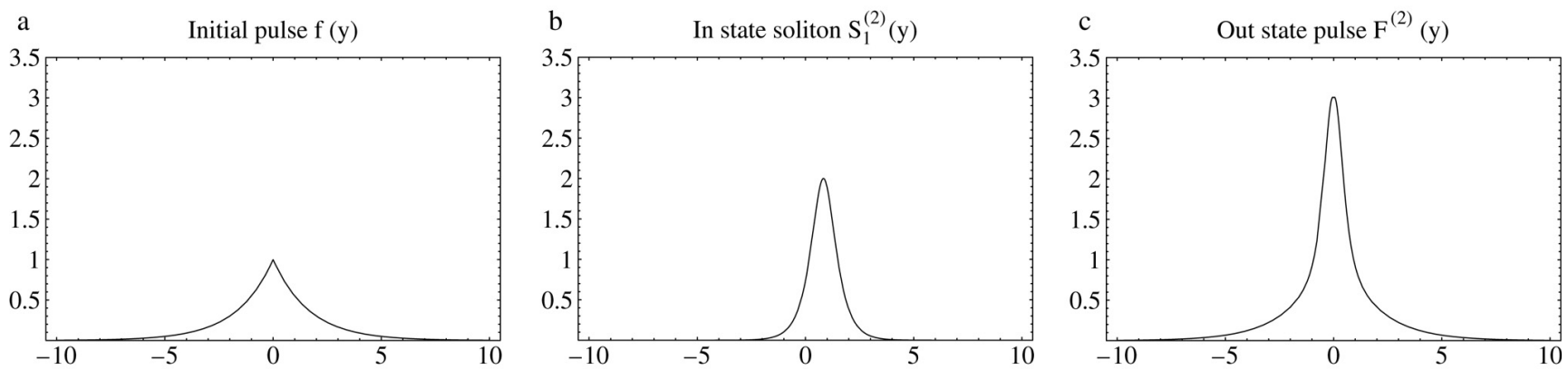

Fig. 3. Width-narrowing and amplitude-amplification.

and with two solitons (for $j=2$, see Figs. 2 and 3) on the exponential picked pulse $f(y)$ (67) when such desirable effects as amplitude-amplification and width-narrowing of the initial in-state profile $f(y)$ occur. In all figures the modulus is plotted.

Fig. 1 refers to the case $j=1$, see the asymptotic states (57a), with the following values of the parameters: $c_{1}=-1, c_{2}=0$, $c_{3}=10, \rho=0.1, \eta=-1, a=2, q=1, \gamma_{1}=\gamma_{2}=\gamma_{3}=1$. In particular, Fig. 1a shows the initial pulse $f(y)$, Fig. $1 \mathrm{~b}$ shows the shape $S_{2}^{(1)}(y)$, see (59a), of the in-state soliton and Fig. 1c shows the out-state pulse $F^{(1)}(y)$, see (58). Figs. 2 and 3 refer instead to the case $j=2$; see the asymptotic states (57b). Here two contra-propagating equally shaped solitons with speeds $c_{1}=-1$ and $c_{3}=1$ collide with the standing $\left(c_{2}=0\right)$ initial pulse $f(y)$.

In Fig. 2, with the following values of the parameters: $\rho=0.1, \eta=1, a=1, q=1, \gamma_{1}=\gamma_{2}=\gamma_{3}=1$, it is shown the case in which the amplitudes and widths of both the two solitons and the pulse $f(y)$ coincide, while in Fig. 3, with the following values of the parameters: $\rho=0.1, \eta=2, a=-1, q=0.6, \gamma_{1}=\gamma_{2}=\gamma_{3}=1$, the amplitude, respectively the width, of the colliding solitons is larger, respectively narrower, than that of the initial pulse $f(y)$.

In particular, Figs. 2a and 3a show the initial pulse $f(y)$, Figs. $2 \mathrm{~b}$ and $3 \mathrm{~b}$ show the common shape of the initial solitons $S_{1}^{(2)}(y)$ and $S_{3}^{(2)}(y)$ (see (57b) and (59b)) while Figs. $2 \mathrm{c}$ and $3 \mathrm{c}$ display the out-state profile $F^{(2)}(y)$; see (58). Figures in the case $j=3$ are not reported since these collisions feature properties similar to those found in the case $j=1$.

We end this section with the observation that our formulae given here are applicable to several other choices of the bare wave $f(y)$ to yield explicit expressions of solutions of the 3WRI equation, provided, of course, that the ZS equations (44) be exactly solvable. Indeed our choice here, i.e. $f(y)=a \exp (-q|y|)$, merely indicates how the interested reader may perform simple numerical experiments with explicit analytic expressions. 


\section{Conclusion and remarks}

The system (1) is a model equation for the resonant interaction of three waves. It has been introduced by the method of multiscale perturbation, as applied to a nonlinear dispersive wave equation, with the purpose of describing the time evolution of the amplitudes $\chi_{1}, \chi_{2}, \chi_{3}$ of three plane waves with wave-numbers $k_{1}, k_{2}, k_{3}$ and frequencies $\omega_{1}, \omega_{2}, \omega_{3}$ in the resonance conditions $k_{1}+k_{2}+k_{3}=0$ and $\omega_{1}+\omega_{2}+\omega_{3}=0$. This system is therefore a rather universal model and it finds itself in several physical applications. Its integrable version, as characterized by the conditions (3), has been therefore actively investigated by means of the theory of solitons. In particular explicit solutions have been obtained which correspond to the purely discrete spectrum, i.e. the multi-soliton solutions. They may be obtained by repeated application of the DDT (see Section 2) to the trivial solution $\chi_{1}=\chi_{2}=\chi_{3}=0$. Here we have applied the DDT to a seed solution with only two vanishing wave amplitudes while the third one is a nonvanishing arbitrary function, and we have thereby constructed a larger class of exact solutions of (1) outside the class of pure discrete spectrum solutions. The feasibility of such construction is due to two special properties of the 3WRI system, namely its lack of dispersion and of selfinteraction. The computational technique is elementary and mainly algebraic. Only one step, namely solving the Zakharov-Shabat system (44), requires analytical skills. In this respect we should point out that a similar approach to construct explicit solutions has already been discussed in [9] in the differential geometric context of $n$ waves depending on $n$ independent variables (and therefore no spectral parameter appears). However, we should notice that that approach in the present context (i.e. three waves depending on two independent variables) would require "solving" the differential equations (44) by giving a priori the value of the spectral parameter $\lambda$ and one of the two "wave-functions", for instance $\phi_{1}(y)$, and deriving therefore $\phi_{2}(y, \lambda)$ and $f(y)$. This approach, which arbitrarily assigns the "wave-functions" rather than the "potential", is certainly easier than the one adopted here but it has drawbacks as regard to physical applications. One inconvenience is that the incoming wave-profile $f(y)$ would depend on $\lambda$ with the implication that no separate control of the parameter $\lambda$ (which is related to the shape of the solitons in the in- and out-states) and of the bare profile $f(y)$ would be possible in numerical experiments. In order to avoid this drawback we prefer to assign $f(y)$ within the (rather large) class of "solvable potentials", rather than the "wave-function" $\phi_{1}(y)\left(\right.$ or $\left.\phi_{2}(y)\right)$ and to solve therefore the equations (44) for any $\lambda$.

We also note that we have confined our construction to the class of localized solutions. However, our formulae apply as well to the case in which the nonvanishing undressed wave $f(y)$, see (40), is outside the class of localized, or finite-energy, wave-profiles. The investigation of these "quasi kink" type solutions is left to future work. One remarkable example of such solutions has been recently displayed in [7]. The soliton solutions introduced there correspond to those obtained by dressing, via the method of Section 2, the function $f(y)=a$, $a$ being an arbitrary complex constant (this case may be viewed also as the $q \rightarrow 0$ (singular) limit of our present choice (67)). These solutions of the 3WRI equation (1) describe the resonant interaction of two "bright" solitons with one "kink" soliton, a process which features various behaviours, such as those of boomerons and trappons or the creation and annihilation of pairs of bright solitons in the background of a kink. The method of DDT described here yields a rather large class of new solutions of the 3WRI equation whose generic behavior is of boomeronic type, depending on particular choices of the undressed solution. This is indeed the first appearance of boomerons as solutions of a model system of PDEs of such wide applicability as the 3WRI equation. In this respect, it should be also pointed out that several systems of coupled Nonlinear Schrödinger type equations which possess solutions with similar boomeronic phenomenology have been recently found and investigated in [8].

\section{Acknowledgments}

Part of this work has been done while attending the Scientific Gathering on Integrable Systems (October 16-December 11 2004) at the Centro International de Ciencias, Cuernavaca, Mexico, and we thank it for partial financial support. The work of SL has been partially supported by a grant from Il Circolo, Italian Cultural Association, London, UK.

\section{References}

[1] V.E. Zakharov, S.V. Manakov, Resonant interaction of wave packets in nonlinear media, Sov. Phys. JETP Lett. 18 (1973) $243-245$.

[2] D.J. Kaup, A. Reiman, A. Bers, Space-time evolution of nonlinear three-wave interactions. I. Interaction in a homogeneous medium, Rev. Modern Phys. 51 (1979) 275-309.

[3] F. Calogero, Universality and integrability of the nonlinear PDEs describing N wave interactions, J. Math. Phys. 30 (1989) 28-40.

[4] F. Calogero, A. Degasperis, Spectral Transform and Solitons, vol. 1, North Holland Pub., Amsterdam, 1982; M.J. Ablowitz, P.A. Clarkson, Solitons, Nonlinear Evolution Equation and Inverse Scattering Transform, in: London Mathematical Society Lecture Note Series, vol. 149, Cambridge University Press, 1991.

[5] V.E. Zakharov, S.V. Manakov, S.P. Novikov, L.P. Pitajevski, The Theory of Solitons: The Inverse Problem Method, Nauka, Moskow, 1980 (in Russian); V.B. Matveev, M.A. Salle, Darboux Transformations and Solitons, Springer-Verlag, Berlin, 1991; C. Rogers, W.K. Schief, Bäcklund and Darboux Transformations, Cambridge University Press, 2002.

[6] M. Abramowitz, I.A. Stegun, Handbook of Mathematical Functions with Formulas, Graphs, and Mathematical Tables, 9th printing, Dover Publishing, New York, 1972.

[7] F. Calogero, A. Degasperis, Novel solution of the system describing the resonant interaction of three waves, Physica D 200 (2005) $242-256$.

[8] F. Calogero, A. Degasperis, New integrable equations of nonlinear Schrödinger type, Stud. Appl. Math. 113 (2004) 91-137.

[9] V. Zakharov, Application of inverse scattering method to problems of differential geometry, Contemp. Math. 301 (2002) 15-33. 